\title{
Interpretation of the emergency electrocardiogram by junior hospital doctors
}

Department of Cardiology, Ninewells Hospital, Dundee, United Kingdom N D Gillespie $S$ D Pringle

A\&E Department, Dundee Royal Infirmary, Dundee, United Kingdom C T F Brett

W G Morrison

Correspondence to: Dr N Gillespie, Department of Cardiology, Ninewells Hospital and Medical School, Dundee DD1 9SY, Scotiand.

Accepted for publication 17 July 1996

\begin{abstract}
Objective-To assess the ability of a cohort of junior hospital doctors to interpret ECGs which have immediate clinical relevance and influence subsequent management of patients

Methods-57 junior hospital doctors were interviewed and asked to complete a standard questionnaire which included eight ECGs for interpretation and a supplementary question relating to the administration of thrombolytic treatment. Each doctor was assessed over a $48 \mathrm{~h}$ period while they performed their daily clinical duties.

Results-The major abnormality of anterior myocardial infarction was recognised by almost all doctors. There was difficulty in the interpretation of posterior myocardial infarction and second degree heart block. Most myocardial infarctions would have been given satisfactory thrombolysis, but there was a reluctance to use this treatment in patients with posterior myocardial infarction and left bundle brach block. A few patients without myocardial infarction would have received thrombolytic treatment.

Conclusions-There is varying ability among junior hospital doctors in the interpretation of the emergency electrocardiogram. The results are of concern as poor interpretation of the ECG can result in inappropriate management. As a result of the findings of this study it is proposed to introduce more formal training in the interpretation of clinically relevant ECG abnormalities for junior hospital doctors. (F Accid Emerg Med 1996;13:395-397)
\end{abstract}

Key terms: ECG interpretation; junior hospital doctors; accident and emergency

The treatment of acute myocardial infarction by thrombolysis results in significant improvement in mortality. ${ }^{12}$ The major clinical trials which have shown benefit from the administration of thrombolytic treatment used electrocardiographic (ECG) criteria to allocate patients to treatment. Recent audits have shown poor ability to interpret ECGs among hospital doctors. ${ }^{3}$ However, these audits have been concentrated on ECG abnormalities of limited clinical importance in the acute medical emergency situation. We assessed the ability of a group of hospital doctors to interpret clinically important ECGs, where accurate assessment was essential for optimum patient management.

\section{Methods}

The study was performed in the departments of accident and emergency, medicine, and surgery in a teaching hospital. Fifty seven medical staff were selected at random and provided with a questionnaire containing eight ECGs for interpretation. Each subject was interviewed by one of the investigators. The study was performed over a $48 \mathrm{~h}$ period, and to prevent circulation of the correct answers those participating were asked not to divulge the answers of the questionnaire to colleagues. The ECGs offered for interpretation had one major clinically significant abnormality from among the following: (1) acute anterior myocardial infarction, (2) atrial flutter with variable heart block, (3) left bundle branch block, (4) atrial fibrillation with a slow ventricular response, (5) posterior myocardial infarction, (6) second degree heart block, (8) supraventricular tachycardia. There was also a normal ECG.

The doctors included in the survey were selected as being the most likely to be interpreting large numbers of ECGs in emergency admissions. We asked the doctors: (1) to describe the relevant ECG abnormalities, and (2) which ECGs had indications for thrombolysis, given a normal previous ECG and a $2 \mathrm{~h}$ history of cardiac chest pain suggestive of myocardial infarction. The study was performed during the working day while the respondents were performing their normal clinical duties. Model answers were provided by two consultant cardiologists.

\section{Results}

The major findings of the study are summarised in tables 1 and 2. Most of the doctors recognised acute anterior myocardial infarction with ease. This is reassuring, as these are the patients most likely to gain overall benefit from the administration of thrombolytic treatment. However, there was difficulty in recognising posterior myocardial infarctions. This is of concern as many of these patients have large infarcts and thrombolysis is often given in the presence of ST depression. The other ECG which proved difficult to interpret was second degree heart block. In practice, this is an important abnormality which should be recognised early as patients may require cardiac pacing if this arrhythmia is associated with haemodynamic upset.

A consistent finding was that thrombolysis would have been given appropriately to patients with anterior myocardial infarction. However, there was a reluctance to give throm- 
Table 1 Percentage of hospital doctors correctly identifying ECG abnormalities

\begin{tabular}{lll}
\hline & \multicolumn{2}{l}{ Department/grade } \\
\cline { 2 - 3 } ECG & $\begin{array}{l}f H O \\
(n=26)\end{array}$ & $\begin{array}{l}\text { Med SHO/Reg+AEE } \\
(n=31)\end{array}$ \\
\hline Anterior MI & 88 & 97 \\
Atrial flutter & 73 & 84 \\
LBBB & 77 & 87 \\
Normal & 73 & 97 \\
Slow AF & 42 & 55 \\
Posterior MI & 8 & 29 \\
2:1 block & 19 & 45 \\
SVT & 69 & 81 \\
\hline
\end{tabular}

JHO, junior house officer; SHO, senior house officer; Med, medical; Reg, registrar; MI, myocardial infarction; LBBB, left bundle branch block; AF, atrial fibrillation; SVT, supraventricular tachycardia.

bolysis to patients with posterior infarctions and those with new left bundle branch block.

A few doctors would have given thrombolysis to patients with atrial fibrillation, second degree heart block, and supraventricular tachycardia.

\section{Discussion}

Electrocardiography is one of the most frequently used investigations in hospital practice. Despite this, previous studies have suggested a poor ability among doctors to interpret the findings. ${ }^{34}$ This is alarming as the optimal management of a number of potentially life threatening conditions requires prompt accurate interpretation of the electrocardiogram. Some of these studies have analysed interpretation of ECG abnormalities such as the PR interval and QT interval which, although important, have little influence on the administration of emergency treatment.

This present study focuses on ECG abnormalities which have immediate implications for patient management. Each ECG had only one major abnormality, and the scoring system was such that the interpretation was either correct or incorrect, thus eliminating the contribution of non-specific ECG findings.

The study confirmed a satisfactory interpretation and treatment of acute anterior myocardial infarction. Posterior myocardial infarction was identified poorly. Even though the established benefit of thrombolysis in posterior myocardial infarction is less clear, key management includes treatment with aspirin, $\beta$ blockade, and admission to a coronary care unit. $^{5}$ The poor recognition of patients with second degree heart block is of particular

Table 2 Percentage of patients thrombolysed given $2 h$ history of chest pain

\begin{tabular}{lll}
\hline & \multicolumn{2}{l}{ Department/grade } \\
\cline { 2 - 3 } & $\begin{array}{l}f H O \\
(n=26)\end{array}$ & Med SHO/Reg+AGE $(n=31)$ \\
\hline Anterior MI & 92 & 97 \\
LBBB & 31 & 39 \\
Posterior & 15 & 23 \\
Slow AF & 12 & 10 \\
SVT/2nd degree heart block & 8 & 3 \\
\hline
\end{tabular}

JHO, junior house officer; SHO, senior house officer; Med, medical; Reg, registrar; MI, myocardial infarction; LBBB, left bundle branch block; AF, atrial fibrillation; SVT, supraventricular tachycardia. importance as these patients are at risk of syncope and may require pacemaker insertion. Our data suggest that there are gaps in the knowledge of junior doctors regarding the indications for thrombolysis. All of the patients with acute anterior myocardial infarction were given thrombolysis but only $40 \%$ of those with left bundle branch block (LBBB) would have received it. This is concerning as there is an absolute mortality benefit of 49 lives saved per 1000 patients treated if patients with LBBB on their presenting ECG are treated with thrombolysis. $^{6}$

Several patients with atrial fibrillation, second degree heart block, and supraventricular tachycardia would have received thrombolysis incorrectly in this study. This highlights the problem that in the desire to give thrombolysis to patients with acute myocardial infarction other patients may receive inappropriate thrombolysis. This exposes these patients to the risk of serious adverse effects such as cerebrovascular accident or major blood loss without any potential for benefit.

The study confirms that there is a varying ability among junior hospital doctors in interpreting ECGs showing myocardial infarction or life threatening arrhythmias. Although in practice doctors with difficulties in interpreting ECGs are likely to get a second opinion before giving thrombolytic treatment there is nevertheless a need for formal teaching in ECG interpretation in the same way that resuscitation skills are taught, as delays in giving thrombolysis have survival implications for patients. ${ }^{7}$ Computer models may be of some help but there is no substitute for clear recognition of abnormalities that require immediate therapeutic intervention. There is a clear need for hospitals to issue guidelines for thrombolysis and standard protocols to aid inexperienced junior staff in decision making. A recent survey of the variation in the use of thrombolytic treatment by junior hospital doctors showed that less than a third of participants worked in hospitals with local policies. ${ }^{8}$ Ideally thrombolytic treatment should be given in accident and emergency departments so that the door-to-needle time is kept below $30 \mathrm{~min}$ utes. $^{7}$ In this region, decisions regarding thrombolytic therapy are made by both medical and accident and emergency staff and treatment is started in accident and emergency.

Clearly continuing education is vital, as there is a rapid turnover of junior hospital doctors in most hospitals. ALS (advanced life support) schemes offer training in basic cardiovascular emergencies and life support, although places on such schemes are limited. Only two of the doctors questioned (both in $\mathrm{A} / \mathrm{E}$ ) in this survey were known to have ALS certification. In the near future, there are plans to introduce an induction programme for all junior house officers before they start their jobs. This programme would include ECG interpretation. Following introduction of this programme it is planned to repeat this study.

In conclusion, this survey shows a wide variation in junior doctors' ability to interpret clinically important ECGs and to give throm- 
bolytic treatment appropriately. The widespread introduction of ALS courses and the adoption of local guidelines may improve training and guidance for junior hospital doctors in a key area of clinical practice.

1 Gruppo Italiano Per Lo Studio Dela Streptokinase in Infarto Miocardico (GISSI). Effectiveness of intravenous thrombolytic treatment in acute myocardial infarction. Lancet 1986; i:397-401.

2 ISIS-2 Collaborative group. Randomised intravenous streptokinase, oral aspirin, both among 17,187 cases of suspected acute myocardial infarction. ISIS-2. Lancet 1988;ii:349-60.
3 Montgomery $\mathbf{H}$, Hunter S, Morris S, Naunton-Morgan R, Marshall RM. Interpretation of electrocardiograms by doctors. BMF 1994;309:1551-2.

4 Morrison WG. Swann IJ. Electrocardiograph interpretation by junior doctors. Arch Emerg Med 1990;7:108-10.

5 Frishman WH, Furberg CD, Friedewald WT. $\beta$ Blockade for survivors of acute myocardial infarction. $N$ Engl $\mathcal{F}$ Med 1984;310:830-4.

6 Indications for fibrinolytic therapy in suspected acute myocardial infarction: collaborative overview of early mortality and major morbidity results from all randomised trials of over 1000 patients. FTT Collaborative Group Lancet 1994;ii:311-22.

7 Guidelines for the early management of patients with myocardial infarction. British Heart Foundation working cordial infarction. Brish Heart Foundation working

8 Hood S, Birnie D, Curzio J, Hillis WS. Wide variation in the use of thrombolytic therapy among junior doctors in South use of thrombolytic therapy among junior doctors in South
and Central Scotland. Health Bull (Edinb) 1996;4:131-9.

\section{ADVANCED LIFE SUPPORT GROUP}

Major Incident Medical Management and Support Courses: to be held in various centres throughout the UK in 1997. This is a three day course in "life support style" designed to train health service personnel to provide an effective response at a major incident.

Fee $£ 300.00$

Advanced Paediatric Life Support Courses: to be held in various centres throughout the UK in 1997. This is a three day course designed to provide training which will enable doctors and nurses to deal efficiently with all paediatric emergencies. The course is modular and has sections on paediatric resuscitation, serious illness and serious injury.

Fee varies according to centre. Range $£ 300.00-£ 350.00$.

Inquiries for both courses to:

Jenny Antrobus

Advanced Life Support Group

Second Floor, The Dock Office,

Trafford Rd., Salford Quays,

Manchester M5 2XB

Tel 01618771999

Fax 01618771666

see panel on page 430 